\title{
Topology and perturbation theory
}

\author{
J.Manjavidze ${ }^{a}$
}

November 10, 2018

\begin{abstract}
Paper contains description of the fields nonlinear modes successive quantization scheme. It is shown that the path integrals for absorption part of amplitudes are defined on the Dirac ( $\delta$-like) functional measure. This permits arbitrary transformation of the functional integral variables. New form of the perturbation theory achieved by mapping the quantum dynamics in the space $W_{G}$ of the (action, angle)-type collective variables. It is shown that the transformed perturbation theory contributions are accumulated exactly on the boundary $\partial W_{G}$. Abilities of the developed formalism are illustrated by the Coulomb problem. This model is solved in the $W_{C}=($ angle, angular momentum, Runge-Lentz vector $)$ space and the reason of its exact integrability is 'emptiness' of $\partial W_{C}$.
\end{abstract}

PACS numbers: 02.30.Cj, 03.65.Db, 02.40.Vh, 31.15.Kb

\section{Introduction}

Solution of great number of the modern field-theoretical problems rest on absence of workable perturbation theory in the vicinity of actions nontrivial extremum $u_{c}(x)$. The think is that the dynamics of perturbations in such fields is rather complicate ${ }^{1}$. So, for instance, beyond the semiclassical approximation of path integrals one should know solution of the equation:

$$
\left(\partial^{2}+v^{\prime \prime}\left(u_{c}\right)\right)_{x} G\left(x, x^{\prime} ; u_{c}\right)=\delta\left(x-x^{\prime}\right)
$$

for the Green function $G\left(x, x^{\prime} ; u_{c}\right)$. The exact solution of this equation is unknown since the operator $\left(\partial^{2}+v^{\prime \prime}\left(u_{c}\right)\right)_{x}$ is not translationally invariant if $u_{c}=u_{c}(x)$. Of course, one can find $G\left(x, x^{\prime} ; u_{c}\right)$ perturbatively neglecting in the first approximation the coordinate dependence in $v^{\prime \prime}\left(u_{c}\right)$. But this approximation is applicable at small distances $\left|x-x^{\prime}\right| \rightarrow 0$ only and the number of modern speculations on the way as this restriction may be avoided is enormous. The perturbative QCD is an example of such solution.

A suspicion that the fields are not always useful variables arise and an idea that the quantum theory formulated in other terms may be much more effective seems natural (actually hoping that the substitution may considerably simplify calculation of the integral). 
I wish to show the quantitative realization of this idea and will construct corresponding perturbation theory. The main formal problem on this way ${ }^{2}$ consist in demonstration that the transformation to new variables is unitary, i.e. conserves the total probability.

So, the main goal of this paper is to formulate the perturbation theory formalism for the case of nontrivial $u_{c}(x)$. Our perturbation theory is nothing new if the field $u_{c}=$ const., but is extremely effective for nontrivial $u_{c}(x)$. Actually the successive approach to the strong-coupling perturbation theory is offered.

Having in mind the non-perturbative effects (field topological excitations) the lattice decompositions are widely used. For instance, number of problems of quantum mechanics was solved using the 'time sliced' method ${ }^{3,4}$. This approach presents the path integral as a finite product of well defined ordinary Cauchy integrals and, therefore, allows perform arbitrary transformations. But transformed 'effective' Lagrangian gains additional term $\sim \hbar^{2}$ in the continuum limit. Last one crucially depends from the way as the 'slicing' was performed and a general solution of this problem is cumbersome. Our approach will not contain any ambiguities.

We will formulate the approach, risking to loose generality, considering the simplest quantum-mechanical examples of particle motion in the potential hole $v(u)$ with one nondegenerate minimum at $u=0$. We will calculate the probability $\rho=\rho(E)$ to find the bound state with energy $E$. Namely, at the end we would solve the plane Coulomb problem using our method.

Our experience may be useful for quantization of nonlinear waves also. Indeed, introducing the convenient variables (collective coordinates) one can reduce the quantum soliton-like excitations problem to quantum-mechanical one. This idea was considered previously by many authors ${ }^{5,6}$.

The aim of this paper is to show

(i) Origin of desired perturbation theory.

The mechanism of unitary, i.e. the total probability conserved, mapping $(u, p)(t) \rightarrow$ $(\xi, \eta)(t)$, where $p(t)$ is the conjugate to $u(t)$ momentum, of the functional measure on the space $W_{G}$ with local coordinates $(\xi, \eta)(t)$ is shown. It would be considered as the factor space $W_{G}=G / \bar{G}$, where $G$ is the theory symmetry group and its subgroup $\bar{G}$ is the symmetry of classical fields $u_{c}=u_{c}(\xi, \eta)$.

It is well known that if $J_{i}=J_{i}(u, p), i=1,2, \ldots, N$ are the first integrals in involution then the canonical transformation $(u, p) \rightarrow(J, Q)$ solves the mechanical problem (Liouville-Arnold theorem). The $(u, p)_{c}$ flow is defined by the $2 N$ system of coupled algebraic equations

$$
\eta=J(u, p), \xi=Q(u, p)
$$

The mapping (2):

$$
J: T \rightarrow W_{G}
$$

where $T$ is the $2 N$-dimensional phase space and $W_{G}$ is a linear space, introduces integral manifold $J_{\omega}=J^{-1}(\omega)$ in such a way that the classical phase space flaw belongs to $J_{\omega}$ completely. 
Our methodological idea assumes quantization of the $J_{\omega}$ manifold instead of flow in $T$. This becomes possible iff the quantum trajectory completely belongs to $J_{\omega}$. Last one means that eqs.(2) have unique solution $(u, p)_{c}$ and $(\xi, \eta)$ compose a manifold.

The 'direct' mapping (3) assumes that $J$ is known. But this approach to general quantum problems seems inconvenient having in mind the nonlinear modes quantization, when the number of degrees of freedom $N=\infty$, or if the transformation is not canonical, see (68). We will consider by this reason the 'inverse' approach starting from assumption that the classical flow exist. Then we would be able to reconstruct the motion in $W_{G}$ since $(u, p)_{c}$ belongs to $W_{G}$ completely.

In other words, we would like to describe the quantum dynamics in the space of classical fields (orbits) $u_{c}$ parameters $(\xi, \eta)(t)$. The unitarity of such mapping is guaranteed be the fact that the functional measure, as the consequence of the unitarity condition, is Diracian ${ }^{7}$ :

$$
D M(u, p)=\delta\left(E-H_{T}(u, p)\right) \prod_{t} d u(t) d p(t) \delta\left(\dot{u}+\frac{\partial H_{j}}{\partial p}\right) \delta\left(\dot{p}-\frac{\partial H_{j}}{\partial u}\right),
$$

where the Hamiltonian

$$
H_{j}=\frac{1}{2} p^{2}+v(u)-j u
$$

includes the energy of quantum fluctuations $j u$, with the provoking quantum excitations force $j=j(t)$. Then the dynamical equilibrium between ordinary mechanical forces $(\dot{p}(t)$, $\left.-v^{\prime}(u)\right)$ and quantum force $(j(t))$ determined by $\delta$-like measure (1) allows to perform arbitrary transformation of quantum measure, i.e. of $j$, caused by transformations of $u$ and $p$.

A theory on such measure is 'simple' since the functional $\delta$-function defines the complete set of contributions. So, the constructive definition of the vacuum will be offered in Sec.IIC. It will be shown the general method of the mapping, applicable for field theories also, when $u_{c}=u_{c}(\vec{x} ; \xi, \eta)$.

Note, there are in modern physics the remarkable attempts to construct a geometrical approach to quantum mechanics ${ }^{8,9}$ and field theory ${ }^{10}$. Our approach has an evident geometrical interpretation and it will be widely used. It has deal with the excitations of classical phase-space flows. By this reason, in contrast with above mentioned approaches, the finite-dimensional manifolds only, as in classical mechanics, would arise even in the field theories.

We would construct the mapping using the base of symplectic geometry. Starting from assumption that $(\xi, \eta)$ form the symplectic space of arbitrary dimension we would demonstrate its projection on $W_{G}$.

Describing the perturbations of new dynamical variables $(\xi, \eta)(t)$ we take into account the quantum excitations of field $u_{c}=u_{c}(\xi, \eta)$. In considered below Coulomb problem the set $(\xi, \eta)=$ (angle, angular momentum, length of Runge-Lentz vector) unambiguously defines the Kepler orbits. Hence, the mapping $(u, p) \rightarrow(\xi, \eta)$ is rightful since the quantum trajectory covers $W_{G}=(\xi, \eta)$ densely (fluctuations of $j(t)$ are defined on Gauss measure) and since $(u, p)_{c}$ belong to $W_{G}$ completely. 
(ii) Structure of perturbation theory in the $G / \bar{G}$ space and as it can be applied.

It will be shown that the quantum corrections of the transformed theory are accumulated on the boundaries (bifurcation manifolds ${ }^{11}$ ) $\partial W_{G}$ of the factor space, i.e. are defined mainly by $W_{G}$ topology. The important quantitative consequence would be the observation that the quantum corrections may disappeared (totally or partly) on $\partial W_{G}$ if the boundary is empty.

So, the problem of quantum corrections we reduce up to definition of intersection of the boundary set $\left\{\partial u_{c}(\xi, \eta)\right\}$ with the boundary $\partial W_{G}$. This circumstance would be useful for estimation of quantum corrections. For all that the explicit form of $u_{c}$ is not necessary since $\left\{\partial u_{c}\right\} \cap \partial W_{G}$ is estimated.

One should assume that $j(t)$ switched on adiabatically (in this case we expand contributions in the vicinity of $j=0$ ) for effective use of this definition of measure. Otherwise we should know $j(t)$ exactly, including it into Lagrangian as the external field. The measure would remain $\delta$-like in last case also. So, the measure (4) allows to conclude that the solutions of classical equation

$$
\frac{\delta S(u)}{\delta u(t)}=0
$$

defines the complete set of contributions ${ }^{12}$.

Eq.(5) reflects the ordinary Hamiltonian variational principle. But the measure (4) contains following additional information:

i. Only strict solutions of eq.(5) should be taken into account.

ii. $\rho(E)$ is described by the sum of all solutions of eq.(5), independently from the value of corresponding fluctuations;

iii. $\rho(E)$ did not contain the interference terms from various topologically nonequivalent contributions. This displays the orthogonality of corresponding Hilbert spaces;

iv. The measure (4) includes $j(t)$ as the 'external source';

$\mathbf{v}$. In the frame of above adiabaticity condition the field $u(t)$ disturbed by $j(t)$ belongs to the same manifold (topology class) as the classical field defined by (5) ${ }^{12}$.

One must underline that the measure (4) is derived for real - time processes only, i.e. is not valid for tunneling ones. By this reason above conclusions should be taken carefully. The corresponding selection rule will be given below in Sec.IIC.

The main results of this paper looks as follows.

(A) If the amplitude has the path integral representation (22), then the unitarity condition leads to following representation for $\rho(E)$

$$
\rho(E)=\int_{0}^{\infty} d T e^{-i \hat{K}(e j)} \int D M e^{-i U(u, e)} e^{-i S_{0}(u)},
$$

where the exponential over differential operator $\hat{K}(e j)$, see (29), gives perturbation series, functional $U(u, e)$, see (27), describes interactions, the measure $D M$ is Diracian, see (4) and $S_{0}$ is the closed path action. 
(B) If coordinate variable $u_{c}(\xi, \eta)$ and corresponding momentum $p_{c}(\xi, \eta)$ obey the equations

$$
\left\{u_{c}, h_{j}\right\}=\frac{\partial H_{j}}{\partial p_{c}},\left\{p_{c}, h_{j}\right\}=-\frac{\partial H_{j}}{\partial u_{c}}, \text { at } j=0,
$$

where $\{$,$\} is the Poisson bracket in the (\xi, \eta)$ space, if

$$
h_{j}(\xi, \eta)=H_{j}\left(u_{c}, p_{c}\right), h(\eta) \equiv h_{0}(\xi, \eta),
$$

where

$$
H_{j}(u, p)=\frac{1}{2} p^{2}+v(u)-j u
$$

is the total Hamiltonian, then (a) the transformed measure has the form:

$$
D M(\xi, \eta)=\delta(E-h(T)) \prod_{t} \delta\left(\dot{\xi}-\frac{\partial h_{j}}{\partial \eta}\right) \delta\left(\dot{\eta}+\frac{\partial h_{j}}{\partial \xi}\right),
$$

since $\left(u_{c}, p_{c}\right)$ are the solutions of incident (classical) Hamiltonian equations, (b) the dimensions of vectors $\left(u_{c}, p_{c}\right)$ and of the space $(\xi, \eta)=W_{G}$ are arbitrary. This property is important since the physical trajectory $u_{c}$ may occupy the space of dimension $\operatorname{dim} W_{G} \leq \operatorname{dim} T$, where $T$ is the incident phase space. Moreover, (c) $\operatorname{dim} W_{G}$ may be even or odd.

(C) If the Green function $g\left(t-t^{\prime}\right)$ of equations

$$
\dot{\xi}=\frac{\partial h_{j}}{\partial \eta}, \dot{\eta}=-\frac{\partial h_{j}}{\partial \xi}
$$

have the form:

$$
g\left(t-t^{\prime}\right)=\theta\left(t-t^{\prime}\right), g(0)=1,
$$

then the quantum corrections to semiclassical approximation are accumulated on the boundaries of $W_{G}$ :

$$
\rho^{q}=\int_{\partial W_{G}} d \rho^{q} .
$$

This conclusion proves $\mathbf{v}$. The explicit form of $d \rho^{q}$ will be given below.

The generalization of formalism on the field theory, where $u_{c}=u_{c}(\vec{x} ; \xi, \eta)$, becomes evident noting (ii) and that the space coordinate may be considered as the index (of special cell). By the same reason (ii) and taking into account (iii) the formalism allows to consider also the situation where $(\xi, \eta)=(\xi, \eta)(x, t)$. Last one incorporates the gauge freedom. So, in result, the mapping allows quantize the gauge theories without Faddeev-Popov ansatz. This is important for non-Abelian gauge theories, where the Faddeev-Popov ghosts and the Gribov's ambiguities present the problem.

The field theories will be considered in subsequent publications. In this paper I wish consider following questions.

- Sec.2. The differential measure for $\rho(E)$ is derived. The connection between unitarity condition and d'Alembert's variational principle is discussed. It is shown that our representation restores the ordinary WKB perturbation series. The main general consequences of functional Dirac measure are listed. 
- Sec.3. The transformations of the path-integral variables are shown. The main purpose is to demonstrate the $(u, p) \rightarrow(\xi, \eta)$ canonical transformation. The coordinate transformations is demonstrated also.

- Sec.4. The main properties of new perturbation theory in the invariant subspace (factor manifold) $W_{G}$ are shown considering the simplest quantum-mechanical example. One can hope that this properties stay useful for field-theoretical models also.

- Sec.5. We solve the Coulomb problem to show explicitly the role of the reduction for quantum systems as the consequence of $(\mathbf{A}, \mathbf{B})$ and $(\mathbf{C})$.

\section{Unitarity condition}

Purpose of this section is to show how the $S$-matrix unitarity condition can be introduced into the path-integral formalism to find measure (4) ${ }^{7}$.

The unitarity condition for the $S$-matrix $S S^{+}=I$ presents the infinite set of nonlinear operator equalities:

$$
i A A^{*}=A-A^{*}
$$

where $A$ is the amplitude, $S=I+i A$. Note, in this definition $A$ is dimensionless. (Obviously the energy-momentum conservation $\delta$-functions are extracted from elements of $S$-matrix and then the net ones have the dimension of space $[x]$ ). Expressing the amplitude by the path integral one can see that the l.h.s. of equality (14) offers the double integral and, at the same time, the r.h.s. is linear combination of integrals. Let us consider what this linearization of product $A A^{*}$ gives.

Using the spectral representation of one-particle amplitude:

$$
A_{1}\left(u_{1}, u_{2} ; E\right)=\sum_{n} \frac{\Psi_{n}^{*}\left(u_{2}\right) \Psi_{n}\left(u_{1}\right)}{E-E_{n}+i \varepsilon}, \quad \varepsilon \rightarrow+0,
$$

let us calculate

$$
\rho(E)=\int d u_{1} d u_{2} A_{1}\left(u_{1}, u_{2} ; E\right) A_{1}^{*}\left(u_{1}, u_{2} ; E\right) .
$$

The integration over end points $u_{1}$ and $u_{2}$ is performed for sake of simplicity only. Using ortho-normalizability of the wave functions $\Psi_{n}(u)$ we find that

$$
\rho(E)=\sum_{n}\left|\frac{1}{E-E_{n}+i \varepsilon}\right|^{2}=\frac{\pi}{\varepsilon} \sum_{n} \delta\left(E-E_{n}\right) .
$$

Certainly, the last equality is nothing new but it is important to note that $\rho(E) \equiv 0$ for all $E \neq E_{n}$, i.e. that all unnecessary contributions with $E \neq E_{n}$ were canceled by difference in the r.h.s. of eq.(14). We will put just last equality in (17) in the basis of the approach.

We will build the perturbation theory for $\rho(E)$ using the path-integral definition of amplitudes ${ }^{7}$. It leads to loss of some information since the amplitudes can be restored in such formulation with the phase accuracy only. Yet, that is quite enough for calculation of the energy spectrum. So, instead of $\operatorname{Sp}\{1 /(E-H+i \varepsilon)\}^{13}$, as follows from (17), the absorption parts $\sim \Im \operatorname{Sp}\{1 /(E-H+i \varepsilon)\}$ would be calculated only. 
The statement that the unitarity condition unambiguously determines the measure of path integral for $\rho(E)$ looks like a tautology since $\exp \{i S(u)\}$, where $S(u)$ is the action, is the unitary operator, which shifts a system along the trajectory. (It is well known that this unitary transformation is the analogy of tangent transformations of classical mechanics ${ }^{14}$.) I.e. the unitarity is already included in the path integrals.

But the general path-integral solution contains unnecessary degrees of freedom (unobservable states with $E \neq E_{n}$ in our example). Our idea is to define the functional measure in such a way that the condition of absence of unnecessary contributions be loaded from the very beginning. Just in this case the unitarity becomes the sufficient condition. Indeed, it will be shown that the equality (14) leads to $\delta$-like functional measure, which unambiguously determines the complete set of classically permitted contributions.

Formal realization is simple: one should find, as it follows from (17), the linear pathintegral representation for $\rho(E)$. Indeed, to see the integral form of our approach, let us use the proper-time representation:

$$
A_{1}\left(u_{1}, u_{2} ; E\right)=\sum_{n} \Psi_{n}\left(u_{1}\right) \Psi_{n}^{*}\left(u_{2}\right) i \int_{0}^{\infty} d T e^{i\left(E-E_{n}+i \varepsilon\right) T}
$$

and insert it into (16):

$$
\rho(E)=\sum_{n} \int_{0}^{\infty} d T_{+} d T_{-} e^{-\left(T_{+}+T_{-}\right) \varepsilon} e^{i\left(E-E_{n}\right)\left(T_{+}-T_{-}\right)} .
$$

We will introduce new time variables instead of $T_{ \pm}$:

$$
T_{ \pm}=T \pm \tau
$$

where, as follows from Jacobian of transformation, $|\tau| \leq T, 0 \leq T \leq \infty$. But we can put $|\tau| \leq \infty$ since $T \sim 1 / \varepsilon \rightarrow \infty$ is essential in integral over $T$. In result,

$$
\rho(E)=2 \pi \sum_{n} \int_{0}^{\infty} d T e^{-2 \varepsilon T} \int_{-\infty}^{+\infty} \frac{d \tau}{\pi} e^{2 i\left(E-E_{n}\right) \tau} .
$$

In the last integral all contributions with $E \neq E_{n}$ are canceled. Note that the product of amplitudes $A A^{*}$ was 'linearized' after extraction of 'virtual' time $\tau=\left(T_{+}-T_{-}\right) / 2$. The physical meaning of such variables will be discussed, see also ${ }^{14}$. I.e. we would divide the dynamical degrees of freedom on the 'classical' (like $T=\left(T_{+}+T_{-}\right) / 2$ ) and 'quantum' (like $\tau$ ) ones. Such decomposition becomes possible if the double integrals are considered.

\subsection{Dirac functional measure}

We will consider following path-integral:

$$
A_{1}\left(u_{1}, u_{2} ; E\right)=i \int_{0}^{\infty} d T e^{i E T} \int_{u_{1}=u(0)}^{u_{2}=u(T)} D x e^{i S_{C_{+}}(u)}
$$


where $C_{+}$is the Mills complex time contour ${ }^{15}$ :

$$
C_{ \pm}: t \rightarrow t \pm i \varepsilon, \varepsilon \rightarrow+0,0 \leq t \leq T_{ \pm}
$$

Calculating the probability to find a particle with energy $E$ ( $\Im E$ is not mentioned for sake of simplicity) we have:

$$
\begin{array}{r}
\rho(E)=\int d u_{1} d u_{2}|A|^{2}=\int_{0}^{\infty} d T_{+} d T_{-} e^{i E\left(T_{+}-T_{-}\right)} \times \\
\int_{u_{+}(0)=u_{-}(0)}^{u_{+}\left(T_{+}\right)=u_{-}\left(T_{-}\right)} D_{C_{+}} u_{+} D_{C_{-}} u_{-} e^{i S_{C_{+}\left(T_{+}\right)}\left(u_{+}\right)-i S_{C_{-}\left(T_{-}\right)}\left(u_{-}\right)},
\end{array}
$$

where, see (23), $C_{-}(T)=C_{+}^{*}(T)$. Note that the total action $S_{\wp}(u) \equiv\left(S_{C_{+}\left(T_{+}\right)}\left(u_{+}\right)-\right.$ $\left.S_{C_{-}\left(T_{-}\right)}\left(u_{-}\right)\right)$describes the closed-path motion by definition.

New independent time variables $T$ and $\tau$ will be used again, see (20). The mean trajectory $u(t)=\left(u_{+}(t)+u_{-}(t)\right) / 2$ and the deviation $e(t)$ from it will be introduced, $u_{ \pm}(t)=u(t) \pm e(t)$. Note, we assume that this linear transformations in the path integrals may be performed.

We will consider $e(t)$ and $\tau$ as the fluctuating, virtual, quantities and calculate the integrals over them perturbatively. In the zero order over $e$ and $\tau$, i.e. in the semiclassical approximation, $u$ is the classical path and $T$ is the total time of classical motion.

The boundary conditions (see (24)) states the closed-path motion. We would consider the boundary conditions for $e(t)$ only:

$$
e(0)=e(T)=0
$$

Note the uniqueness of this solution if the integral over $\tau$ is calculated perturbatively.

Extracting the linear over $e$ and $\tau$ terms from the closed-path action $S_{\wp}$ and expanding over $e$ and $\tau$ the remainder terms:

$$
-\tilde{H}_{T}(u ; \tau)=\left(S_{C_{+}(T+\tau)}(u)-S_{C_{-}(T-\tau)}(u)\right)+2 \tau H_{T}(u)-S_{0}(u),
$$

where $H_{T}(u)$ is the Hamiltonian at the time moment $T$, and

$$
-U_{T}(u, e)=\left(S_{C_{+}(T)}(u+e)-S_{C_{-}(T)}(u-e)\right)+2 \Re \int_{C_{+}(T)} d t\left(\ddot{u}+v^{\prime}(u)\right) e
$$

we find that

$$
\rho(E)=2 \pi \int_{0}^{\infty} d T e^{-i \hat{K}(\omega, \tau ; j, e)} \int D M(u) e^{-i \tilde{H}_{T}(u ; \tau)-i U_{T}(u, e)+i S_{0}(u)} .
$$

Note the necessity of boundary condition (25) to find (28). It allows to split the expansions over $\tau$ and $e$.

The expansion over differential operators:

$$
\hat{K}(\omega, \tau ; j, e)=\frac{1}{2}\left(\frac{\partial}{\partial \omega} \frac{\partial}{\partial \tau}+\Re \int_{C_{+}(T)} d t \frac{\delta}{\delta j(t)} \frac{\delta}{\delta e(t)}\right)
$$


will generate the perturbation series. We will assume that it exist at least in Borel sense. In (28) the functional measure

$$
D M(u)=\delta\left(E+\omega-H_{T}(u)\right) \prod_{t} d u(t) \delta\left(\ddot{u}+v^{\prime}(u)-j\right)
$$

unambiguously defines the complete set of contributions in the path integral. The functional $\delta$-function is defined as follows:

$$
\prod_{t} \delta\left(\ddot{u}+v^{\prime}(u)-j\right)=(2 \pi)^{2} \int_{e(0)=0}^{e(T)=0} \prod_{t} \frac{d e(t)}{\pi} e^{-2 i \Re \int_{C_{+}} d t e\left(\ddot{x}+v^{\prime}(u)-j\right)}
$$

Note, the phase in (31) stay real for arbitrary directions in the complex plane of $e$. This explains why calculation of the modulo square of amplitudes is important.

The physical meaning of this $\delta$-function is following. We can consider $\left(\ddot{u}+v^{\prime}(u)-j\right)$ as the total force and $e(t)$ as the virtual deviation from true trajectory $u(t)$. In classical mechanics the virtual work must be equal to zero: $\left(\ddot{u}+v^{\prime}(u)-j\right) e(t)=0$ (d'Alembert)

16 since the motion is time reversible. From this evident dynamical principle one can find the 'classical' equation of motion:

$$
\ddot{u}+v^{\prime}(u)=j
$$

since $e(t)$ is arbitrary.

In quantum theories the virtual work usually is not equal to zero, i.e. the quantum motion is not time reversible since the quantum corrections can shift the energy levels. But integration over $e(t)$, with boundary conditions (25), leads to the same result (32). So, in quantum theories the unitarity condition ${ }^{7}$ play the same role as the d'Alembert's variational principle in classical mechanics. We can conclude, the unitarity condition as the dynamical principle establish the time - local equilibrium between classical (1.h.s. of (32)) and quantum (r.h.s. of (32)) forces.

So, considering the double integral we may introduce integration over two independent fields $u$ and $e$. Then, (i) integral over $e$ gives the $\delta$-function (31) and (ii) last one defines integral over $u$. This definition of path integrals permits the Mills' analitical continuation into complex time plane.

It should be underlined that the real-time field theory is considered. We found actually that the real-time theories are simple, see the functional measure (1). This seems important since the Wick rotation is practically noncontrollable if the symmetry is high (symmetry content of a theory is sensitive to the space-time metrics ${ }^{17}$ ) and especially if the dynamical problems are solved.

\subsection{Comparison with WKB perturbation theory}

Let us consider now the representation (28). It is not hard to show that it restores the perturbation theory of stationary phase method. For this purpose it is enough to consider the ordinary integral:

$$
A(a, b)=\int_{-\infty}^{+\infty} \frac{d x}{(2 \pi)^{1 / 2}} e^{i\left(\frac{1}{2} a x^{2}+\frac{1}{3} b x^{3}\right)}
$$


with $\Im a \rightarrow+0$ and $b>0$. Computing the 'probability' $\rho=|A|^{2}$ we find:

$$
\rho(a, b)=e^{\frac{1}{2 i} \hat{j} \hat{e}} \int_{-\infty}^{+\infty} d x e^{-2\left(x^{2}+e^{2}\right) \operatorname{Im} a} e^{2 i \frac{b}{3} e^{3}} \delta\left(\Re a x+b x^{2}+j\right) .
$$

The 'hat' symbol means, as usual, the derivative over corresponding quantity: $\hat{X} \equiv \partial / \partial X$. One should put the auxiliary variables $(j, e)$ equal to zero at the very end of calculations.

Performing the trivial transformation $e \rightarrow i e, \hat{e} \rightarrow-i \hat{e}$ of auxiliary variable we find in the limit $\Im a=0$ that the contribution of $x=0$ extremum (minimum) gives expression:

$$
\rho(a, b)=\frac{1}{a} e^{-\frac{1}{2} \hat{j} \hat{e}}\left(1-4 b j / a^{2}\right)^{-1 / 2} e^{2 \frac{b}{3} e^{3}}
$$

and the expansion of operator exponent gives the asymptotic series:

$$
\rho(a, b)=\frac{1}{a} \sum_{n=0}^{\infty}(-1)^{n} \frac{(6 n-1) ! !}{n !}\left(\frac{2 b^{4}}{3 a^{6}}\right)^{n}, \quad(-1) ! !=0 ! !=1 .
$$

This series is convergent in Borel sense.

Note, if $|A|$ only is interesting for us then eq.(34) may be considered as the definition of integral (33). By this reason one may put $\Im a=0$ from the very beginning. We will consider this suggestion more carefully.

Let us calculate now $\rho$ using the stationary phase method. Contribution from the minimum $x=0$ gives $(\Im a=0)$ :

$$
A(a, b)=e^{-i \hat{j} \hat{x}} e^{-\frac{i}{2 a} j^{2}} e^{i \frac{b}{3} x^{3}}\left(\frac{i}{a}\right)^{1 / 2} .
$$

The corresponding 'probability' is

$$
\rho(a, b)=\frac{1}{a} e^{-\frac{1}{2} \hat{j} \hat{e}} e^{2 \frac{b}{3} e^{3}} e^{\frac{2 b}{a^{2}} e j^{2}}
$$

This expression does not coincide with (35) but it leads to the same asymptotic series (36).

To find the representation (37) from (35) the transformation

$$
\delta\left(\Re a x+b x^{2}+j\right)=e^{-\frac{i}{2} \hat{j}^{\prime} \hat{e}^{\prime}} e^{+2 i\left(b x^{2}+j\right) e^{\prime}} \delta\left(\Re a x+j^{\prime}\right)
$$

can be applied. Indeed, inserting this equality into (35) we find (37). The eq. 38) is evident from the Fourier transformation of $\delta$-function.

Note, the transformation (38) practically solves, linearizing argument of $\delta$-function, the problem of computation of the determinant. This will be important considering functional integrals. Moreover, it reflects the freedom in choice of terms in which the perturbation theory in vicinity of nontrivial trajectories in functional space is realized.

Just this property is the source of splitting:

$$
j(t) \rightarrow\left(j_{\theta}(t), j_{h}(t)\right)
$$

of the 'Lagrange' source $j(t)$ onto set of sources to each independent degree of freedom of the invariant subspace if the transformation (A) was performed. This splitting is demonstrated in Appendix A. By this way the actually Hamiltonian description is achieved in the invariant subspace. 


\subsection{General properties of theory with $\delta$-like measure}

The solution $x_{j}(t)$ of eq.(32) we would search expanding it over $j(t)$ :

$$
u_{j}(t)=u_{c}(t)+\int d t_{1} G\left(t, t_{1} ; u_{c}\right) j\left(t_{1}\right)+\ldots
$$

This is sufficient since $j(t)$ is the auxiliary variable. In this decomposition $u_{c}(t)$ is the strict solution of unperturbated equation $\ddot{x}+v^{\prime}(x)=0$ and $G\left(t, t^{\prime} ; u_{c}\right)$ must obey eq.(11). Note that the functional $\delta$-function in (31) does not contain the end-point values of time $t=0$ and $t=T$. This means that the initial conditions to the eq.(32) are not fixed and the integration over them should be performed because of our definition of $\rho$.

The $\delta$-likeness of measure allows to conclude that all strict regular solutions (including trivial) of classical (unperturbated by $j$ ) equation(s) of motion must be taken into account.

We must consider only 'strict' solutions because of strict cancellation of needless contributions. The $\delta$-likeness of measure means that the probability $\rho(E)$ should contain a sum over all discussed solutions. This is the main distinction of our unitary method of quantization from stationary phase method: even having few solution there is not interference terms in the sum over them in $\rho$. Note that the interference terms are absent independently from solutions 'nearness' in the functional space. This reflects the orthogonality of Hilbert spaces builded on the various $u_{c}{ }^{1}$ and is the consequence of unitarity condition.

The solutions must be regular since the singular $u_{c}$ gives zero contribution on $\delta$-like measure.

It is evident that having the sum over contributions of various $u_{c}$ we must leave largest. This selection rule ${ }^{7}$ is the constructive definition of physical vacuum.

Summation over all solutions of classical equation of motion means also necessity to take into account all topologically-equivalent orbits $u_{c}$. This means integration over the volume $V_{W}$ of factor space $W_{G}=G / \bar{G}$. This naturally introduces integration over zeromode degrees of freedom.

So, our selection rule looks as follows: if there is not special external constraints then in the sum over topologically nonequivalent trajectories one should leave, up to the volume $V_{W}$, the contribution defined in the highest factor manifold $G / \bar{G}$ if $G$ is the theory symmetry group and $\bar{G}$ is the $u_{c}$-invariance (sub)group of $G$ group. (Note, $G$ may be wider then the actions invariance group.) This selection rule means that $\bar{G}$ should be the lowest (sub)group.

Note, the quantum corrections may violate our selection rule.

The Dirac measure defines the real-time motion only and is not applicable for tunnelling processes since reflects the dynamical equilibrium of 'real' forces. The contributions from tunneling processes should be added to the contributions defined by our $\delta$-like measure. Then, following to our selection rule, we should leave those contribution(s) which are proportional to the highest volume $V_{W}$. So, our definition of measure is rightful if the real-time contributions factor manifold have the highest dimension. One can say in this case that the imaginary-time contributions are realized on zero measure $\left(\sim 1 / V_{W}\right)$. 
The explicit investigation of this condition is the nontrivial task in spite of its seeming simplicity (the dimension of $G / \bar{G}$ is defined by classical solution only). Actually we should know all classical orbits and the quantum corrections may 'shrink' the dimension of $W_{G}$. So, above selection rule gives the classification only of mostly probable contributions. Following from our selection rule we should start from the nontrivial solutions $u_{c} \neq 0$ since the volume of trivial $u_{c}=0$ is equal to zero.

\section{Canonical transformations}

It is evident that the measure (30) admits the canonical transformations (the coordinate transformations are described in Appendix B). This follows from $\delta$-likeness of measure. The phase space differential measure has the form:

$$
D M(x, p)=\delta\left(E+\omega-H_{T}(u)\right) \prod_{t} d u d p \delta\left(\dot{u}-\frac{\partial H_{j}}{\partial p}\right) \delta\left(\dot{p}+\frac{\partial H_{j}}{\partial u}\right),
$$

where

$$
H_{j}=\frac{1}{2} p^{2}+v(u)-j u
$$

is the time dependent through $j(t)$ total Hamiltonian.

The transformation may be performed inserting

$$
1=\int D \theta D h \prod_{t} \delta\left(h-\frac{1}{2} p^{2}-v(u)\right) \delta\left(\theta-\int^{u} d u(2(h-v(u)))^{-1 / 2}\right) .
$$

It is important that both differential measures in (42) and (40) are $\delta$-like. This allows to change the order of integration and firstly integrate over $(u, p)$. Calculating result one can use the $\delta$-functions of (40). In this case the $\delta$-functions of (42) will define the constraints. But if we use the $\delta$-functions of (42) the mapping $(u, p) \rightarrow(\theta, h)$ is performed and the remaining $\delta$-functions would define motion in the factor space $W_{G}$. We conclude that our transformation takes into account the constraints since both ways must give the same result. Note also, the transformation did not change the power of manifolds since both measures, in $T$ and in $T^{*} G$, are $\delta$-like.

We find by explicit calculations that:

$$
D M(\theta, h)=\delta(E+\omega-h(T)) \prod_{t} d \theta d h \delta\left(\dot{\theta}-\frac{\partial h_{j}}{\partial h}\right) \delta\left(\dot{h}+\frac{\partial h_{j}}{\partial \theta}\right),
$$

since considered transformation is canonical, $\{h(u, p), \theta(u, p)\}=1$, where

$$
h_{j}(\theta, h)=h-j u_{c}(\theta, h)
$$

is the transformed Hamiltonian and $u_{c}(\theta, h)$ is the classical trajectory parametrized by $h$ and $\theta$. 


\subsection{General properties of the transformed perturbation theory}

The transformed perturbation theory presents expansion over $1 / g$ if $u_{c} \sim 1 / g$, where $g$ is the interaction constant. Hence, we construct the perturbation theory in the 'strong coupling' limit. But one should remind also that, generally, all solutions must be taken into account. This means that the perturbation theory for $\rho(E)$ contains simultaneously both series over $g$ (from trivial solution $u_{c}=0$ ) and over $1 / g$, i.e. the sum of weekcoupling and strong-coupling expansions. According to our selection rule we should leave largest among them, i.e. start consideration from contributions of nontrivial trajectories.

In the invariant subspace $W_{G}$ we must solve following equations of motion:

$$
\dot{h}=j \frac{\partial u_{c}(\theta, h)}{\partial \theta}, \quad \dot{\theta}=1-j \frac{\partial u_{c}(\theta, h)}{\partial h} .
$$

They have a simple structure: the 'propagator' in $W_{G}$ space is simple $\Theta$-function.

Indeed, expanding solutions of eqs. 45) over $j$, the zero order solutions are $\theta_{0}=t_{0}+t$ and $h_{0}=$ const. The first order over $j$ gives:

$$
\dot{h}_{1}\left(t, t^{\prime}\right)=\delta\left(t-t^{\prime}\right) \frac{\partial u_{c}\left(\theta_{0}, h_{0}\right)}{\partial \theta_{0}}, \quad \dot{\theta}_{1}\left(t, t^{\prime}\right)=-\delta\left(t-t^{\prime}\right) \frac{\partial u_{c}\left(\theta_{0}, h_{0}\right)}{\partial h_{0}} .
$$

This leads to the first order equation for Green function $g\left(t-t^{\prime}\right)$ :

$$
\dot{g}\left(t-t^{\prime}\right)=\delta\left(t-t^{\prime}\right) .
$$

The solution of this equation introduces the time 'irrevercibility':

$$
g\left(t-t^{\prime}\right)=\Theta\left(t-t^{\prime}\right)
$$

in opposite to causal particles propagator $G\left(t, t^{\prime} ; u_{c}\right)$. But, as will be seen below, the perturbation theory with Green function (47) is time reversible. Note also, that the solution (47) is the unique and is the direct consequence of usual in the quantum theories $i \varepsilon$-prescription.

The uncertainty is contained in the boundary value $g(0)$. We will see that $g(0)=0$ excludes number of quantum corrections. By this reason one should consider $g(0) \neq 0$. We will assume that

$$
g(0)=1
$$

since this boundary condition to eq. (46) is natural for local theories. We will use also following formal equalities:

$$
g\left(t-t^{\prime}\right) g\left(t^{\prime}-t\right)=0, \quad 1=g\left(t-t^{\prime}\right)+g\left(t^{\prime}-t\right)
$$

considering $g\left(t-t^{\prime}\right)$ as the distribution (generalized function).

Note, the important property (13) of our perturbation theory is the consequence of boundary condition (48). 
The property $\Im g(t)=0$ on the real time axis allows to conclude that the perturbation theory in the $W_{G}$ space can be constructed on the real-time axis. This excludes natural for probabilistic description doubling of degrees of freedom. But, for more confidence, one should introduce the $i \varepsilon$-prescription and, extracting the $\delta$-function in the measure, to analyze the theory boundary conditions in $\varepsilon=0$ limit. We will return to this question at the end of this section.

\subsection{Splitting of Lagrange source $j(t)$}

Note now that $j \partial u_{c} / \partial \theta$ and $j \partial u_{c} / \partial h$ in the r.h.s. of (45) can be considered as the new (renormalized) sources. This allows to note that the mapping on the $W_{G}$ splits 'Lagrange' quantum force $j$ on a set of quantum forces individual to each independent degree of freedom.

Indeed, the simple algebra gives (see Appendix A):

$$
\begin{array}{r}
\rho(E)=2 \pi \int_{0}^{\infty} d T e^{\frac{1}{2 i}\left(\hat{\omega} \hat{\tau}+\Re \int_{C_{+}} d t\left(\hat{j}_{h}(t) \hat{e}_{h}(t)+\hat{j}_{\theta}(t) \hat{e}_{\theta}(t)\right)\right)} \\
\times \int D h D \theta e^{-i \tilde{H}\left(u_{c} ; \tau\right)-i V_{T}\left(u_{c}, e_{c}\right)+i S_{0}\left(u_{c}\right)} \\
\times \delta(E+\omega-h(T)) \prod_{t} \delta\left(\dot{h}-j_{h}\right) \delta\left(\dot{\theta}-1-j_{\theta}\right),
\end{array}
$$

where

$$
e_{c}=e_{h} \frac{\partial u_{c}}{\partial \theta}-e_{\theta} \frac{\partial u_{c}}{\partial h} \equiv\left(e_{h} \hat{\theta}-e_{\theta} \hat{h}\right) u_{c} .
$$

Note, $e_{c}$ carry the symplectic structure of Hamiltonian equations of motion, see (43), i.e. $e_{c}$ is the invariant of canonical transformations.

Hiding the $u_{c}(t)$ dependence in $e_{c}$ we solve the problem of the functional determinants and simplify the equation of motion as much as possible. Performing the shift:

$$
\theta \rightarrow \theta+\theta^{\prime}, \quad h \rightarrow h+h^{\prime}
$$

where

$$
\theta^{\prime}(t)=\int_{0}^{T} d t^{\prime} g\left(t-t^{\prime}\right) j_{\theta}\left(t^{\prime}\right), \quad h^{\prime}(t)=\int_{0}^{T} d t^{\prime} g\left(t-t^{\prime}\right) j_{h}\left(t^{\prime}\right)
$$

we can consider $\left(\theta^{\prime}, h^{\prime}\right)$ as the independent virtual variables:

$$
D M(h, \theta)=\delta\left(E+\omega-h(T)-h^{\prime}(T)\right) \prod_{t} d h(t) d \theta(t) \delta(\dot{h}(t)) \delta(\dot{\theta}(t)-1)
$$

and new perturbations generating operator takes the form:

$$
\hat{K}=\frac{1}{2}\left(\hat{\omega} \hat{\tau}+\int_{0}^{T} d t_{1} d t_{2} \Theta\left(t_{2}-t_{1}\right)\left(\hat{e}_{h}\left(t_{1}\right) \hat{h}^{\prime}\left(t_{2}\right)+\hat{e}_{\theta}\left(t_{1}\right) \hat{\theta}^{\prime}\left(t_{2}\right)\right) .\right.
$$

In $U_{T}\left(u_{c}, e_{c}\right)$ we must change $h \rightarrow\left(h+h^{\prime}\right)$ and $\theta \rightarrow\left(\theta+\theta^{\prime}\right)$. 


\subsection{Zero modes problem}

Noting that

$$
\int \prod_{t} d X(t) \delta(\dot{X}(t))=\int d X(0)=\int d X_{0}
$$

we see that the measure (52) coincide with the measure of ordinary integrals over $h_{0}$ and $t_{0}$. Last one defines the volume of translational mode. Note, using naively the WKB expansion we should find $\rho \sim V^{2}$, where $V$ is the zero modes volume, since $\rho \sim|A|^{2}$. But, as follows from (52) and (54), we may find only that $\rho \sim V$. This evident discrepancy follows from our rough analytical continuation on the real time axis: it may, as was noted above, eliminate a doubling of degrees of freedom intrinsic to considered approach.

Let us consider this question more carefully. Deriving explicit form of the operator $\hat{\mathcal{O}}$ following boundary conditions was applied:

$$
u_{+}\left(t \in \partial C_{ \pm}\right)=u_{-}\left(t \in \partial C_{ \pm}\right),
$$

where $\partial C_{ \pm}$are boundaries on corresponding branches of the total Mills time contour $C=C_{+}+C_{-}$. Generally, performing canonical mapping $(u, p) \rightarrow(\theta, h)$,

$$
\left.\left.(u, p)(t)\right|_{C_{ \pm}} \rightarrow(\theta, h)(t)\right|_{C_{ \pm}}
$$

since one should hold the Mills contours memory. Then, noting that the auxiliary variable $e\left(t \in \partial C_{ \pm}\right)=0$, the boundary conditions (55) means following equalities:

$$
u_{c}\left(\theta_{+}, h_{+}\right)\left(t \in \partial C_{ \pm}\right)=u_{c}\left(\theta_{-}, h_{-}\right)\left(t \in \partial C_{ \pm}\right)
$$

Inserting here the explicit value of $u_{c}$ we find the boundary conditions for $\left.(\theta, h)\right|_{ \pm}(t)$. Hence, the doubling of degrees of freedom would disappeared iff (57) leads to equality

of generalized coordinates $(\theta, h)$ on the corresponding boundaries $\partial C_{ \pm}$. Contrary the doubling of degrees of freedom should be taken into account.

We will find solving the equations (52) that the doubling of degrees of freedom should be taken into account in definition of initial data $(\theta(0), h(0))$ only since the Green function of transformed theory is nonsingular on the real time axis. So, if the solution of (57) gives, for instance, $h_{+}(0)=h_{-}(0)$ then the doubling of scale degree of freedom $h(0)$ would disappear. Note also, if the classical trajectory is periodic function then we may choose the initial phases $\sim \theta_{ \pm}(0)$ independently. Just this effect takes into account the phase $S_{0}$ in (26).

From very beginning the measure $D M(u, p)$ is defined on the whole Mills contour $C=C_{+}+C_{-}$:

$$
D M(u, p)=\prod_{t \in C_{+}} \prod_{t \in C_{-}} \ldots
$$

assuming corresponding generalization of $\delta$-functions on the complex arguments, see (31). This property should be conserved in the transformed perturbation theory. Hence, if the boundary condition (57) will not lead to disappearance of the doubling, after integrations we would have, instead of (54), double integrals

$$
\int d X_{+}(0) d X_{-}(0)
$$




\section{Perturbation theory}

Let us consider motion in the action-angle phase space. Corresponding perturbations generating operator has the form:

$$
\hat{K}=\frac{1}{2} \int_{0}^{T} d t d t^{\prime} \Theta\left(t-t^{\prime}\right)\left(\hat{I}(t) \hat{e}_{I}\left(t^{\prime}\right)+\hat{\phi}(t) \hat{e}_{\phi}\left(t^{\prime}\right)\right) \equiv \hat{K}_{I}+\hat{K}_{\phi}
$$

The result of integration using last $\delta$-function is

$$
\rho(E)=2 \pi \int_{0}^{\infty} d T e^{-i \hat{K}} \int_{0}^{2 \pi} \frac{d \phi_{0}}{\omega(E)} e^{-i U_{T}\left(u_{c}, e_{c}\right)},
$$

where

$$
\omega=\partial h\left(I_{0}\right) / \partial I_{0}
$$

and $I_{0}=I_{0}(E)$ is defined by the algebraic equation:

$$
E=h(I) \text {. }
$$

The classical trajectory

$$
u_{c}(t)=u_{c}\left(I_{0}(E)+I(t)-I(T), \phi_{0}+\tilde{\omega} t+\phi(t)\right),
$$

where

$$
\tilde{\omega}=\frac{1}{t} \int_{0}^{T} d t^{\prime} \Theta\left(t-t^{\prime}\right) \omega\left(I_{0}+I\left(t^{\prime}\right)\right) .
$$

The interaction 'potential' $U_{T}$ depends from

$$
e_{c}=e_{\phi} \frac{\partial u_{c}}{\partial I}-e_{I} \frac{\partial u_{c}}{\partial \phi}
$$

The operator (53) contains unnecessary terms. One can omit the $\tau$ dependance since the closed-path motion is described. This simplification was used in (59) and (60).

The operator $\hat{K}$ is linear over $\hat{e}_{\phi}, \hat{e}_{I}$. The result of its action can be written in the form:

$$
\rho(E)=2 \pi \int_{0}^{\infty} d T \int_{0}^{2 \pi} \frac{d \phi_{0}}{\omega(E)}: e^{-i U_{T}\left(u_{c}, \hat{e}_{c} / 2 i\right)} e^{i S_{0}\left(u_{c}\right)}:
$$

where

$$
\hat{e}_{c}=\hat{j}_{\phi} \frac{\partial u_{c}}{\partial I}-\hat{j}_{I} \frac{\partial u_{c}}{\partial \phi}=\left(\hat{j}_{\phi} \hat{I}-\hat{j}_{I} \hat{\phi}\right) u_{c}=\int_{0}^{T} d t^{\prime} \theta\left(t-t^{\prime}\right)\left\{\hat{\phi}\left(t^{\prime}\right), \hat{I}(t)\right\} u_{c}(t)
$$

since

$$
\hat{j}_{X}(t)=\int_{0}^{T} d t^{\prime} \Theta\left(t^{\prime}-t\right) \hat{X}\left(t^{\prime}\right), \quad X=\phi, I .
$$

The colons in (63) means 'normal product': the differential operators must stay to the left of all functions in expansion over commutator

$$
\left\{\hat{\phi}\left(t^{\prime}\right), \hat{I}(t)\right\}=\hat{\phi}\left(t^{\prime}\right) \hat{I}(t)-\hat{I}\left(t^{\prime}\right) \hat{\phi}(t)
$$


Now we are ready to offer the important statement: If the eqs. (47, 48, 49) are hold then each term of perturbation theory in the invariant subspace $W_{G}$ can be represented as the sum of total derivatives over the subspace $W_{G}$ coordinates.

This statement directly follows from definition of perturbation generating operator $\hat{K}$ on the cotangent bundle (53) and of homogeneity of the cotangent manifold in the classical approximation. The proof of this statement is given in Appendix C.

So, we can conclude, contributions are defined by boundary values of classical trajectory $u_{c}$ in the invariant subspace since the integration over $X_{0}=(\xi, \eta)_{0}$ is assumed, see (60), and since contributions are the sum of total derivatives over $X_{0}$.

\section{H-atom problem}

Let us calculate now the integral:

$$
\rho(E)=\int_{0}^{\infty} d T e^{-i \hat{K}(j, e)} \int D M(p, l, r, \varphi) e^{-i U_{T}(r, e)} e^{i S_{0}},
$$

where $\hat{K}(j, e)$ was defined in (B.3) and $D M(p, l, r, \varphi)$ in (B.2).

Considering the Coulomb potential

$$
U_{T}(r, e)=\int_{0}^{T} d t\left[\frac{1}{\left(\left(r+e_{r}\right)^{2}+r^{2} e_{\varphi}^{2}\right)^{1 / 2}}-\frac{1}{\left(\left(r-e_{r}\right)^{2}+r^{2} e_{\varphi}^{2}\right)^{1 / 2}}+2 \frac{e_{r}}{r}\right]
$$

describes the interaction.

We will restrict ourselves by the plane problem. Corresponding phase space $T=$ $(p, l, r, \varphi)$ is 4 -dimensional. But the classical flaw of this problem can be parametrized by the angular momentum $l$, corresponding angle $\varphi$ and by the normalized on total Hamiltonian Runge-Lentz vectors length $n$. So, we will demonstrate the mapping ( $p$ is the radial momentum in the cylindrical coordinates):

$$
J_{l, n}:(p, l, r, \varphi) \rightarrow(l, n, \varphi)
$$

to construct the perturbation theory in the $W_{C}=(l, n, \varphi)$ space. I.e. $W_{C}$ is not the symplectic space: $W_{C}=T^{*} G \times R^{1}$, where $(l, \varphi) \in T^{*} G$ is the symplectic space and $n \in R^{1}$. Nevertheless we start from the symplectic space adding to $n$ the auxiliary canonical variable $\xi$.

It is well known ${ }^{18}$ that the consequence of hidden conservation of the Runge-Lentz vector $\vec{N}$ is closeness of the Kepler orbits independently from initial conditions. In result the orbit is the function of $|\vec{N}|$ only. The external field leads to precession of $\vec{N}$ and the orbit should be parametrized by 4 parameters in this case. So, the reduction (68) takes into account the hidden symmetry of the Coulomb problem.

The bound state energies $(E<0)$ in the Coulomb potential to illustrate our idea will be calculated. This popular problem was considered by many authors, using various methods ${ }^{18}$. The path-integral solution of this problem was offered in ${ }^{19}$ using the timesliced method. 


\subsection{General formalism of mapping}

We would consider now more general method of mapping in the $W_{C}$ space. It is important to start from the assumption that the invariant subspace has symplectic structure of cotangent manifold and its farther possible reduction to linear subspace $W_{C}\left(\operatorname{dim}\left(T^{*} G\right) \leq\right.$ $\left.\operatorname{dim}\left(W_{C}\right) \leq \operatorname{dim}(T)\right)$ would be realized as the reduction of quantum degrees of freedom.

The first step of mapping consist in demonstration that the classical trajectories belong to $T^{*} G$ completely. Let

$$
\Delta=\int \prod_{t} d^{2} \xi d^{2} \eta \delta\left(r-r_{c}(\xi, \eta)\right) \delta\left(p-p_{c}(\xi, \eta)\right) \delta\left(l-l_{c}(\xi, \eta)\right) \delta\left(\varphi-\varphi_{c}(\xi, \eta)\right)
$$

be the functional of some functions $(r, p, \varphi, l)(t)$ and $(\xi, \eta)$ are two-vectors. Introducing this functional we realize the transformation:

$$
(r, p, \varphi, l) \rightarrow(r, p, \varphi, l)_{c}(\xi, \eta)
$$

i.e. we want to 'hide' the $t$ dependence into the four functions $(\xi, \eta)(t)$ introducing the composite functions $(r, p, \varphi, l)_{c}(\xi, \eta)$. This four functions will be defined later. The functions $(\xi, \eta)(t)$ are arbitrary.

So, it is assumed that there exist such functions $(r, p, \varphi, l)(t)$ for given $(r, p, \varphi, l)_{c}$ that

$$
\begin{array}{r}
\Delta_{c}=\int \prod_{t} d^{2} \bar{\xi} d^{2} \bar{\eta} \delta\left(\frac{\partial r_{c}}{\partial \xi} \cdot \bar{\xi}+\frac{\partial r_{c}}{\partial \eta} \cdot \bar{\eta}\right) \delta\left(\frac{\partial p_{c}}{\partial \xi} \cdot \bar{\xi}+\frac{\partial p_{c}}{\partial \eta} \cdot \bar{\eta}\right) \times \\
\delta\left(\frac{\partial \varphi_{c}}{\partial \xi} \cdot \bar{\xi}+\frac{\partial \varphi_{c}}{\partial \eta} \cdot \bar{\eta}\right) \delta\left(\frac{\partial l_{c}}{\partial \xi} \cdot \bar{\xi}+\frac{\partial l_{c}}{\partial \eta} \cdot \bar{\eta}\right) \neq 0
\end{array}
$$

Note that this is the condition for $(r, p, \varphi, l)_{c}(\xi, \eta)$ only.

To perform the mapping we insert

$$
1=\Delta / \Delta_{c}
$$

into (66) and integrate over $(r, p, \varphi, l)(t)$. The proof of equality (71) is following. It assumes that one always can find $(\xi, \eta)$ from four equalities $(r, p, \varphi, l)_{c}(\xi, \eta)=(r, p, \varphi, l)(t)$. Then, noting (70) and using definition of $\delta$-function, eq.(71) becomes evident.

In result of simple calculations (see Appendix D) we find that

$$
D M(\xi, \eta)=\delta\left(E-H_{0}\right) \prod_{t} d^{2} \xi d^{2} \eta \delta^{2}\left(\dot{\xi}-\frac{\partial h_{j}}{\partial \eta}\right) \delta^{2}\left(\dot{\eta}+\frac{\partial h_{j}}{\partial \xi}\right),
$$

where $H_{0}=H_{0}(\eta)$ is the classical Hamiltonian, $H_{0}=H_{j}$ at $j=0$. It is the desired result of transformation of the measure for given 'generating' functions $(r, p, \varphi, l)_{c}(\xi, \eta)$. In this case the 'Hamiltonian' $h_{j}(\xi, \eta)$ is defined by four equations (D.3):

$$
\begin{gathered}
\left\{r_{c}, h_{j}\right\}-\frac{\partial H_{j}}{\partial p_{c}}=0,\left\{p_{c}, h_{j}\right\}+\frac{\partial H_{j}}{\partial r_{c}}=0, \\
\left\{\varphi_{c}, h_{j}\right\}-\frac{\partial H_{j}}{\partial l_{c}}=0,\left\{l_{c}, h_{j}\right\}+\frac{\partial H_{j}}{\partial \varphi_{c}}=0 .
\end{gathered}
$$


But there is another possibility. Let us assume that

$$
h_{j}(\xi, \eta)=H_{j}\left(r_{c}, p_{c}, \varphi_{c}, l_{c}\right)
$$

and the functions $(r, p, \varphi, l)_{c}(\xi, \eta)$ are unknown. Then eqs.(73) are the equations for this functions. It is not hard to see that the eqs.(73) simultaneously with equations given by $\delta$-functions in $(\sqrt{72})$ are equivalent of incident equations if the equality (74) is hold. So, incident dynamical problem was divided on two parts. First one defines the trajectory in the $W_{C}$ space through eqs.(73). Second one defines the dynamics, i.e. the time dependence, through the equations in arguments of $\delta$-functions in the measure (72).

We should consider $r_{c}, p_{c}, \varphi_{c}, l_{c}$ as the classical orbits in the $\xi, \eta$ parametrization. The desired parametrization of them is well known (one can find it in arbitrary textbook of classical mechanics):

$$
r_{c}=\frac{\eta_{1}^{2}\left(\eta_{1}^{2}+\eta_{2}^{2}\right)^{1 / 2}}{\left(\eta_{1}^{2}+\eta_{2}^{2}\right)^{1 / 2}+\eta_{2} \cos \xi_{1}}, p_{c}=\frac{\eta_{2} \sin \xi_{1}}{\eta_{1}\left(\eta_{1}^{2}+\eta_{2}^{2}\right)^{1 / 2}}, \varphi_{c}=\xi_{1}, l_{c}=\eta_{1} .
$$

At the same time,

$$
h_{j}=\frac{1}{2\left(\eta_{1}^{2}+\eta_{2}^{2}\right)^{1 / 2}}-j_{r} r_{c}-j_{\varphi} \varphi_{c} \equiv h(\eta)-j_{r} r_{c}-j_{\varphi} \varphi_{x} .
$$

Note that $\xi_{2}$ is the irrelevant variable for classical flow (75). This conclusion hides the assumption that the space is flat and homogeneous, i.e. an external field may violate this solution.

Our mapping contains two steps. We introduce the set of ansatz functions $(r, p, \varphi, l)_{c}$ assuming that the eqs.(73) have solution $h_{j}(\xi, \eta)$ for arbitrary $j$ and that the condition (70) is hold. For this purpose auxiliary variable $\xi_{2}$ was added assuming that $\partial r_{c} / \partial \xi_{2} \sim \epsilon \rightarrow 0$. In result we found the measure $(\overline{72})$ and

$$
\dot{\xi}=\frac{\partial h_{j}}{\partial \eta}, \dot{\eta}=-\frac{\partial h_{j}}{\partial \xi}
$$

Having (72) we may invert the problem assuming that just $h_{j}$ is known: $h_{j}=H_{j}+O(\epsilon)$, see (74). In this case the eqs.(73) gave $(r, p, \varphi, l)_{c}$ and taking $\partial r_{c} / \partial \xi_{2} \sim \epsilon=0$ this set is the classical flow.

\subsection{Reduction of quantum degrees of freedom}

Noting that the derivatives over $\xi_{2}$ are equal to zero we find that

$$
\begin{aligned}
D M(\xi, \eta)= & \delta(E-h(T)) \prod_{t} d^{2} \xi d^{2} \eta \delta\left(\dot{\xi}_{1}-\omega_{1}+j_{r} \frac{r_{c}}{\partial \eta_{1}}\right) \times \\
& \delta\left(\dot{\xi}_{2}-\omega_{2}+j_{r} \frac{r_{c}}{\partial \eta_{2}}\right) \delta\left(\dot{\eta}_{1}-j_{r} \frac{\partial r_{c}}{\partial \xi_{1}}-j_{\varphi}\right) \delta\left(\dot{\eta}_{2}\right),
\end{aligned}
$$


where

$$
\omega_{i}=\frac{\partial h(\eta)}{\partial \eta_{i}}
$$

are the conserved in classical limit $j_{r}=j_{\varphi}=0$ velocities in the $W_{C}$ space.

It is seen from (78) that the length of Runge-Lentz vector is not perturbated by the quantum forces $j_{r}$ and $j_{\varphi}$. To investigate the consequence of this fact it is necessary to project this forces on the axis of $W_{C}$ space. This means splitting of $j_{r}, j_{\varphi}$ on $j_{\xi}, j_{\eta}$. Then noting that the last $\delta$-function in (78) is source-free, we find the same representation as (66) but with

$$
\hat{K}(j, e)=\int_{0}^{T} d t\left(\hat{j}_{\xi_{1}} \hat{e}_{\xi_{1}}+\hat{j}_{\xi_{2}} \hat{e}_{\xi_{2}}+\hat{j}_{\eta_{1}} \hat{e}_{\eta_{1}}\right),
$$

where the operators $\hat{j}$ are defined by the equality:

$$
\hat{j}_{X}(t)=\int_{0}^{T} d t^{\prime} \theta\left(t-t^{\prime}\right) \hat{X}\left(t^{\prime}\right)
$$

and $\theta\left(t-t^{\prime}\right)$ is the Green function of our perturbation theory.

We should change also

$$
e_{r} \rightarrow e_{c}=e_{\eta_{1}} \frac{\partial r_{c}}{\partial \xi_{1}}-e_{\xi_{1}} \frac{\partial r_{c}}{\partial \eta_{1}}-e_{\xi_{2}} \frac{\partial r_{c}}{\partial \eta_{2}}, \quad e_{\varphi} \rightarrow e_{\xi_{1}}
$$

in the eq.67). The differential measure takes the simplest form:

$$
\begin{array}{r}
D M(\xi, \eta)=\delta(E-h(T)) \prod_{t} d^{2} \xi d^{2} \eta \delta\left(\dot{\xi}_{1}-\omega_{1}-j_{\xi_{1}}\right) \delta\left(\dot{\xi}_{2}-\omega_{2}-j_{\xi_{2}}\right) \\
\times \delta\left(\dot{\eta}_{1}-j_{\eta_{1}}\right) \delta\left(\dot{\eta}_{2}\right) .
\end{array}
$$

Note now that the $\xi, \eta$ variables are contained in $r_{c}$ only: $r_{c}=r_{c}\left(\xi_{1}, \eta_{1}, \eta_{2}\right)$. Then the action of the operator $\hat{j}_{\xi_{2}}$ gives identical to zero contributions into perturbation theory series. And, since $\hat{e}_{\xi_{2}}$ and $\hat{j}_{\xi_{2}}$ are conjugate operators, see (80), we must put $j_{\xi_{2}}=e_{\xi_{2}}=0$. This conclusion ends the reduction:

$$
\begin{aligned}
\hat{K}(j, e) & =\int_{0}^{T} d t\left(\hat{j}_{\xi_{1}} \hat{e}_{\xi_{1}}+\hat{j}_{\eta_{1}} \hat{e}_{\eta_{1}}\right), \\
e_{c} & =e_{\eta_{1}} \frac{\partial r_{c}}{\partial \xi_{1}}-e_{\xi_{1}} \frac{\partial r_{c}}{\partial \eta_{1}} .
\end{aligned}
$$

Using (54) the measure takes the form:

$$
D M(\xi, \eta)=\delta(E-h(T)) d \xi_{2} d \eta_{2} \prod_{t} d \xi_{1} d \eta_{1} \delta\left(\dot{\xi}_{1}-\omega_{1}-j_{\xi_{1}}\right) \delta\left(\dot{\eta}_{1}-j_{\eta_{1}}\right)
$$

since $r_{c}$ is $\xi_{2}$ independent. 


\subsection{Topological analyses}

One can see from (86) that the reduction can not solve the H-atom problem completely: there are nontrivial corrections to the orbital degrees of freedom $\left(\xi_{1}, \eta_{1}\right)$. By this reason we should consider the expansion over $\hat{K}$.

Using last $\delta$-functions in (86) we find, see also ${ }^{12}$ (normalizing $\rho(E)$ on the integral over $\left.\xi_{2}\right)$ :

$$
\rho(E)=\int_{0}^{\infty} d T e^{-i \hat{K}(j, e)} \int d M e^{i S_{0}-i U_{T}\left(r_{c}, e\right)},
$$

where

$$
d M=\frac{d \xi_{1} d \eta_{1}}{\omega_{2}(E)}
$$

The operator $\hat{K}(j, e)$ was defined in (84) and

$$
U_{T}\left(r_{c}, e_{c}\right)=+\int_{0}^{T} d t\left[\frac{1}{\left(\left(r_{c}+e_{c}\right)^{2}+r_{c}^{2} e_{\xi_{1}}^{2}\right)^{1 / 2}}--\frac{1}{\left(\left(r_{c}-e_{c}\right)^{2}+r_{c}^{2} e_{\xi_{1}}^{2}\right)^{1 / 2}}+2 \frac{e_{c}}{r_{c}}\right]
$$

with $e_{c}$ defined in (85) and

$$
r_{c}(t)=r_{c}\left(\eta_{1}+\eta(t), \bar{\eta}_{2}(E, T), \xi_{1}+\omega_{1}(t)+\xi(t)\right),
$$

where $\bar{\eta}_{2}(E, T)$ is the solution of equation $E=h(\eta)$.

The integration range over $\xi_{1}$ and $\eta_{1}$ is as follows:

$$
\partial W_{C}: 0 \leq \xi_{1} \leq 2 \pi, \quad-\infty \leq \eta_{1} \leq+\infty .
$$

First inequality defines the principal domain of the angular variable $\varphi$ and second ones take into account the clockwise and anticlockwise motions of particle on the Kepler orbits, $\left|\eta_{1}\right|=\infty$ is the bifurcation line. Note, this excludes the singularity at $r=0$.

We can write:

$$
\rho(E)=\int_{0}^{\infty} d T \int d M: e^{-i U_{T}\left(r_{c}, \hat{e}\right)} e^{i S_{0}}:
$$

since the operator $\hat{K}$ is linear over $\hat{e}_{\xi_{1}}, \hat{e}_{\eta_{1}}$. The colons means our 'normal product' and $U_{T}\left(r_{c}, \hat{e}\right)$ is the functional of operators:

$$
2 i \hat{e}_{c}=\hat{j}_{\eta_{1}} \frac{\partial r_{c}}{\partial \xi_{1}}-\hat{j}_{\xi_{1}} \frac{\partial r_{c}}{\partial \eta_{1}}, \quad 2 i \hat{e}_{\xi_{1}}=\hat{j}_{\xi_{1}}
$$

Expanding $U_{T}\left(r_{c}, \hat{e}\right)$ over $\hat{e}_{c}$ and $\hat{e}_{\eta_{1}}$ we find:

$$
U_{T}\left(r_{c}, \hat{e}\right)=2 \sum_{n+m \geq 1} C_{n, m} \int_{0}^{T} d t \frac{\hat{e}_{c}^{2 n+1} \hat{e}_{\eta_{1}}^{m}}{r_{c}^{2 n+2}}
$$

where $C_{n, m}$ are the numerical coefficients. We see that the interaction part presents expansion over $1 / r_{c}$ and, therefore, the expansion over $U_{T}$ generates an expansion over $1 / r_{c}$. 
In result,

$$
\rho(E)=\int_{0}^{\infty} d T \int d M\left\{e^{i S_{0}\left(r_{c}\right)}+B_{\xi_{1}}\left(\xi_{1}, \eta_{1}\right)+B_{\eta_{1}}\left(\xi_{1}, \eta_{1}\right)\right\}
$$

The first term is the pure semiclassical contribution and last ones are the quantum corrections. They can be written as the total derivatives:

$$
B_{\xi_{1}}\left(\xi_{1}, \eta_{1}\right)=\frac{\partial}{\partial \xi_{1}} b_{\xi_{1}}\left(\xi_{1}, \eta_{1}\right), \quad B_{\eta_{1}}\left(\xi_{1}, \eta_{1}\right)=\frac{\partial}{\partial \eta_{1}} b_{\eta_{1}}\left(\xi_{1}, \eta_{1}\right)
$$

This means that the mean value of quantum corrections in the $\xi_{1}$ direction are equal to zero:

$$
\int_{0}^{2 \pi} d \xi_{1} \frac{\partial}{\partial \xi_{1}} b_{\xi_{1}}\left(\xi_{1}, \eta_{1}\right)=0
$$

since $r_{c}$ is the closed trajectory independently from initial conditions.

In the $\eta_{1}$ direction the motion is classical:

$$
\int_{-\infty}^{+\infty} d \eta_{1} \frac{\partial}{\partial \eta_{1}} b_{\eta_{1}}\left(\xi_{1}, \eta_{1}\right)=0
$$

since (i) $b_{\eta_{1}}$ is the series over $1 / r_{c}^{2}$ and (ii) $r_{c} \rightarrow \infty$ when $\left|\eta_{1}\right| \rightarrow \infty$. Therefore,

$$
\rho(E)=\int_{0}^{\infty} d T \int d M e^{i S_{0}\left(r_{c}\right)}
$$

This is the desired result.

Noting that

$$
S_{0}\left(r_{c}\right)=k S_{1}(E), \quad k=0, \pm 1, \pm 2, \ldots,
$$

where $S_{1}(E)$ is the action over one classical period $T_{1}$ :

$$
\frac{\partial S_{1}(E)}{\partial E}=T_{1}(E)
$$

and using the identity ${ }^{7}$ :

$$
\sum_{-\infty}^{+\infty} e^{i n S_{1}(E)}=2 \pi \sum_{-\infty}^{+\infty} \delta\left(S_{1}(E)-2 \pi n\right)
$$

we find, normalizing on zero-modes volume, that

$$
\rho(E)=\pi \sum_{n} \delta\left(E+1 / 2 n^{2}\right) .
$$




\section{Conclusion}

Described approach is based on three 'whales'. They are (i) the definition of observables in quantum theories as the modulo square of amplitudes, (ii) the description of quantum processes as the transformation induced by unitary operator $\exp \{i S(x)\}$, where $S(x)$ is the classical action and (iii) the unitarity condition as the principle which determines connection between quantum dynamics and classical measurement (optical theorem). Less principal assumptions, usually taken 'by treaty', that the quantum perturbations are switched on adiabatically, and the Feynman's $i \varepsilon$-prescription, were used also.

The formalism in terms of observables only was considered to use all above fundamental principles. It must be noted that we are forced to work in terms of observables $\rho(E)$ since, this was mentioned above, the transformation mix the degrees of freedom in such a way that it is impossible return to the habitual amplitudes formalism, writing $\rho \sim|A|^{2}$.

Offered approach should be considered as the useful technical trick (probably not unique) helping to calculate the observables if the complicated topologies should be taken into account and the corresponding vacuum is so complicated that its quantitative description is a hopeless task.

\section{Acknowledgement}

I would like to thank my colleagues in the Institute of Physics (Tbilisi), Institute of Mathematics (Tbilisi) and especially A.Ushveridze and I.Paziashvili for helpful discussions. The help of L.Lipatov and E.Levin in realization of the approach was crucial. The paper was compiled in the JINR (Dubna, Russia). I would like to thank V.G.Kadyshevsi for fruitful interest to described technique and underling idea. Further extension on the field theory and possible applications in the particles physics was done with A.Sissakian (see hep-th/9811160) and will be published. The work was partly granted by Georgian Acad. of Sciences.

\section{A Source cotangent foliation}

Let us consider the perturbation-generating operators $\hat{K}$ action to show the splitting mechanism of the source $j(t)$ :

$$
\begin{array}{r}
e^{-i \frac{1}{2} \Re \int_{C_{+}} d t \hat{j}(t) \hat{e}(t)} e^{-i U_{T}\left(u_{c}, e\right)} \prod_{t} \delta\left(\dot{h}-j \frac{\partial u_{c}}{\partial \theta}\right) \delta\left(\dot{\theta}-1+j \frac{\partial u_{c}}{\partial h}\right)= \\
\int D e_{h} D e_{\theta} e^{2 i \Re \int_{C_{+}} d t\left(e_{h} \dot{h}+e_{\theta}(\dot{\theta}-1)\right)} e^{-i U_{T}\left(u_{c}, e_{c}\right)},
\end{array}
$$

where

$$
e_{c}=e_{h} \frac{\partial u_{c}}{\partial \theta}-e_{\theta} \frac{\partial u_{c}}{\partial h} \equiv\left(e_{h} \hat{\theta}-e_{\theta} \hat{h}\right) u_{c}
$$


The integrals over $\left(e_{h}, e_{\theta}\right)$ will be calculated perturbatively:

$$
\begin{array}{r}
e^{-i U_{T}\left(u_{c}, e_{c}\right)}=\sum_{n_{h}, n_{\theta}=0}^{\infty} \frac{1}{n_{h} ! n_{\theta} !} \int \prod_{k=1}^{n_{h}}\left(d t_{k} e_{h}\left(t_{k}\right)\right) \prod_{k=1}^{n_{\theta}}\left(d t_{k}^{\prime} e_{\theta}\left(t_{k}^{\prime}\right)\right) \times \\
P_{n_{h}, n_{\theta}}\left(u_{c}, t_{1}, \ldots, t_{n_{h}}, t_{1}^{\prime}, \ldots, t_{n_{\theta}}\right),
\end{array}
$$

where

$$
P_{n_{h}, n_{\theta}}\left(u_{c}, t_{1}, \ldots, t_{n_{h}}, t_{1}^{\prime}, \ldots, t_{n_{\theta}}\right)=\prod_{k=1}^{n_{h}} \hat{e}_{h}^{\prime}\left(t_{k}\right) \prod_{k=1}^{n_{\theta}} \hat{e}_{\theta}^{\prime}\left(t_{k}^{\prime}\right) e^{-i U_{T}\left(u_{c}, e_{c}^{\prime}\right)}
$$

with $e_{c}^{\prime} \equiv e_{c}\left(e_{h}^{\prime}, e_{\theta}^{\prime}\right)$ and the derivatives in this equality are calculated at $e_{h}^{\prime}=0, e_{\theta}^{\prime}=0$. At the same time,

$$
\begin{array}{r}
\prod_{k=1}^{n_{h}} e_{h}\left(t_{k}\right) \prod_{k=1}^{n_{\theta}} e_{\theta}\left(t_{k}^{\prime}\right)=\prod_{k=1}^{n_{h}}\left(i \hat{j}_{h}\left(t_{k}\right)\right) \prod_{k=1}^{n_{\theta}}\left(i \hat{j}_{\theta}\left(t_{k}^{\prime}\right)\right) \\
\times e^{-2 i \Re \int_{C_{+}} d t\left(j_{h}(t) e_{h}(t)+j_{\theta}(t) e_{\theta}(t)\right)} .
\end{array}
$$

The limit $\left(j_{h}, j_{\theta}\right)=0$ is assumed. Inserting (A.4), A.5) into (A.1) we find new representation for $\rho(E)$ :

$$
\begin{array}{r}
\rho(E)=2 \pi \int_{0}^{\infty} d T e^{i S_{0}\left(u_{c}\right)} e^{\frac{1}{2(}\left(\hat{\omega} \hat{\tau}+\Re \int_{C_{+}} d t\left(\hat{j}_{h}(t) \hat{e}_{h}(t)+\hat{j}_{\theta}(t) \hat{e}_{\theta}(t)\right)\right)} \times \\
\int D h D \theta e^{-i \tilde{H}\left(u_{c} ; \tau\right)-i U_{T}\left(u_{c}, e_{c}\right)} \times \\
\delta(E+\omega-h(T)) \prod_{t} \delta\left(\dot{h}-j_{h}\right) \delta\left(\dot{\theta}-1-j_{\theta}\right)
\end{array}
$$

in which the 'energy' and the 'time' quantum degrees of freedom are splitting.

\section{B Coordinate transformations}

Let us consider the coordinate transformations. For instance, the two dimensional model with potential $v=v\left(\left(x_{1}^{2}+x_{2}^{2}\right)^{1 / 2}\right)$ is simplified considering it in the cylindrical coordinates $x_{1}=r \cos \phi, x_{2}=r \sin \phi$. Note, this transformation is not canonical.

Starting from flat space with trivial metric tensor $g_{\mu \nu}$ and inserting

$$
1=\int \operatorname{Dr} D \phi \prod_{t} \delta\left(r-\sqrt{x_{1}^{2}+x_{2}^{2}}\right) \delta\left(\phi-\arctan \frac{x_{2}}{x_{1}}\right)
$$

we find the measure in the cylindrical coordinates:

$$
\begin{array}{r}
D^{(2)} M(r, \phi)=\delta\left(E+\omega-H_{T}(r, \phi)\right) \times \\
\prod_{t} d r d \phi r^{2}(t) \delta\left(\ddot{r}-\dot{\phi}^{2} r+v^{\prime}(r)-j_{r}\right) \delta\left(\partial_{t}\left(\dot{\phi} r^{2}\right)-r j_{\phi}\right),
\end{array}
$$

where $v^{\prime}(r)=\partial v(r) / \partial r$ and $j_{r}, j_{\phi}$ are the components of $\vec{j}$ in the cylindrical coordinates. 
The perturbation generating operator has the form:

$$
\hat{K}(j, e)=\frac{1}{2}\left\{\hat{\omega} \hat{\tau}+R e \int_{C_{+}} d t\left(\hat{j}_{r}(t) \hat{e}_{r}(t)+\hat{j}_{\phi}(t) \hat{e}_{\phi}(t)\right)\right\}
$$

and in $U_{T}(x, e)$ we must change $e$ on $e_{c}$ with components

$$
e_{c, 1}=e_{r} \cos \phi-r e_{\phi} \sin \phi, \quad e_{c, 2}=e_{r} \sin \phi+r e_{\phi} \cos \phi .
$$

Note, $e_{\phi}$ was arise in product with $r$.

The transformation looks quite classically but the measure (B.2) and perturbation generating operator (B.3) can not be derived by naive coordinate transformation of initial path integral for amplitude. This becomes evident noting that transformed representation for $\rho(E)$ can not be written in the product form $\sim A A^{*}$ of two functional integrals.

It is interesting also to find the measure starting from the curved space with the Lagrangian

$$
L=\frac{1}{2} g_{\mu \nu}(y) \dot{y}^{\mu} \dot{y}^{\nu}-v(y)
$$

It is enough to consider the kinetic term only since, to find the Dirac measure, we should extract the odd over $e$ terms from the 'closed-path' action $S_{T}(y+e)-S_{T}(y-e)$. This procedure is 'trivial' for potential term. The lowest over $e_{\mu}$ part of the kinetic term have the form:

$$
2\left\{g_{\lambda \mu} \ddot{y}^{\mu}+\Gamma_{\lambda, \mu \nu} \dot{y}^{\mu} \dot{y}^{\nu}\right\}
$$

Therefore, the semiclassical approximation is restored.

To find the quantum corrections we should linearize at least the $O\left(e^{3}\right)$ term in the exponent

$$
\exp \left\{\Re \int d t g_{\lambda, \mu \nu} e^{\lambda} \dot{e}^{\mu} \dot{e}^{\nu}\right\}
$$

This is possible noting that

$$
e^{\mu}\left(t^{\prime}\right) \hat{e}_{\mu}^{\prime}\left(t^{\prime}\right) \dot{e}^{\prime \nu}(t)=e^{\mu}\left(t^{\prime}\right) \delta_{\mu \nu} \partial_{t^{\prime}} \delta\left(t-t^{\prime}\right)=\dot{e}^{\nu} \delta\left(t-t^{\prime}\right) .
$$

In result,

$$
D M(y)=\sqrt{|g(y+e)||g(y-e)|} \prod_{\lambda} \prod_{t} d y_{\lambda} \delta\left(g_{\lambda \mu} \ddot{y}^{\mu}+\Gamma_{\lambda, \mu \nu} \dot{y}^{\mu} \dot{y}^{\nu}+v_{\lambda}(y)-j_{\lambda}\right) .
$$

where $v_{\lambda}(y)=\partial_{\lambda} v(y)$ and $\Gamma_{\lambda, \mu \nu}$ is the Christoffel index. The perturbations generating operator $\hat{K}$ and the weight functional $U_{T}(y ; e)$ have the standard form.

\section{Extraction of total derivatives}

By definition $U_{T}$ is the odd over $\hat{e}_{c}$ local functional:

$$
U_{T}\left(u_{c}, \hat{e}_{c}\right)=2 \int_{0}^{T} \sum_{n=1}^{\infty}\left(\hat{e}_{c}(t) / 2 i\right)^{2 n+1} v_{n}\left(u_{c}\right),
$$


where $v_{n}\left(u_{c}\right)$ is some function of $u_{c}$. Inserting (64) we find:

$$
: e^{-i U_{T}\left(u_{c}, \hat{e}_{c}\right)}:=\prod_{n=1}^{\infty} \prod_{k=0}^{2 n+1}: e^{-i U_{k, n}\left(\hat{j}, u_{c}\right)}:
$$

where

$$
U_{k, n}\left(\hat{j}, u_{c}\right)=\int_{0}^{T} d t\left(\hat{j}_{\phi}(t)\right)^{2 n-k+1}\left(\hat{j}_{I}(t)\right)^{k} b_{k, n}\left(u_{c}\right) .
$$

Explicit form of the function $b_{k, n}\left(u_{c}\right)$ is not important.

Using definition (65) it easy to find:

$$
\hat{j}\left(t_{1}\right) b_{k, n}\left(u_{c}\left(t_{2}\right)\right)=\Theta\left(t_{1}-t_{2}\right) \partial b_{k, n}\left(u_{c}\right) / \partial X_{0}
$$

since $u_{c}=u_{c}\left(X(t)+X_{0}\right)$, see (61), or

$$
\hat{j}_{X, 1} b_{2}=\Theta_{12} \partial_{X_{0}} b_{2}
$$

since indices $(k, n)$ are not important.

Let as start consideration from the first term with $k=0$. Then expanding $\hat{U}_{0, n}$ we describe the angular quantum fluctuations only. Noting that $\partial_{X_{0}}$ and $\hat{j}$ commute we can consider lowest orders over $\hat{j}$. The typical term of this expansion is (omitting index $\phi$ )

$$
\hat{j}_{1} \hat{j}_{2} \cdots \hat{j}_{m} b_{1} b_{2} \cdots b_{m}
$$

It is enough to show that this quantity is the total derivative over $\phi_{0}$. The number $m$ counts an order of perturbation, i.e. in $m$-th order we have $\left(\hat{U}_{0, n}\right)^{m}$.

$m=1$. In this approximation we have, see (C.4),

$$
\hat{j}_{1} b_{1}=\Theta_{11} \partial_{0} b_{1}=\partial_{0} b_{1} \neq 0 \text {. }
$$

Here the definition (46) was used.

$m=2$. This order is less trivial:

$$
\hat{j}_{1} \hat{j}_{2} b_{1} b_{2}=\Theta_{21} b_{1}^{2} b_{2}+b_{1}^{1} b_{2}^{1}+\Theta_{12} b_{1} b_{2}^{2}
$$

where

$$
b_{i}^{n} \equiv \partial^{n} b_{i} .
$$

Deriving (C.7) the first equality in (49) was used. At first glance (C.7) is not the total derivative. But inserting

$$
1=\Theta_{12}+\Theta_{21},
$$

(see the second equality in (49)) we can symmetrize it:

$$
\begin{array}{r}
\hat{j}_{1} \hat{j}_{2} b_{1} b_{2}=\Theta_{21}\left(b_{1}^{2} b_{2}+b_{1}^{1} b_{2}^{1}\right)+\Theta_{12}\left(b_{1} b_{2}^{2}+b_{1}^{1} b_{2}^{1}\right)= \\
\partial_{0}\left(\Theta_{21} b_{1}^{1} b_{2}+\Theta_{12} b_{1} b_{2}^{1}\right)
\end{array}
$$


since the explicit form of function $b$ is not important. So, the second order term can be reduced to the total derivative also. Note, that the contribution (C.9) contains the sum of all permutations. This shows the 'time reversibility' of the constructed perturbation theory.

Let us consider now expansion over $\hat{U}_{k, m}, k \neq 0$. The typical term in this case is

$$
\hat{j}_{1}^{1} \hat{j}_{2}^{1} \cdots \hat{j}_{l}^{1} \hat{j}_{l+1}^{2} \hat{j}_{l+2}^{2} \cdots \hat{j}_{m}^{2} b_{1} b_{2} \cdots b_{m}, \quad 0<l<m
$$

where, for instance,

$$
\hat{j}_{k}^{1} \equiv \hat{j}_{I}\left(t_{k}\right), \quad \hat{j}_{k}^{2} \equiv \hat{j}_{\phi}\left(t_{k}\right)
$$

and

$$
\hat{j}_{1}^{i} b_{2}=\Theta_{12} \partial_{0}^{i} b_{2}
$$

instead of (C.4).

$m=2, l=1$. We have in this case:

$$
\begin{array}{r}
\hat{j}_{1}^{1} \hat{j}_{2}^{2} b_{1} b_{2}=\Theta_{21}\left(b_{2} \partial_{0}^{1} \partial_{0}^{2} b_{1}+\left(\partial_{0}^{2} b_{2}\right)\left(\partial_{0}^{1} \partial_{0}^{2} b_{1}\right)\right)+ \\
\Theta_{12}\left(b_{1} \partial_{0}^{1} \partial_{0}^{2} b_{2}+\left(\partial_{0}^{2} b_{2}\right)\left(\partial_{0}^{1} \partial_{0}^{2} b_{1}\right)\right)=\partial_{0}^{1}\left(\Theta_{21} b_{2} \partial_{0}^{2} b_{1}+\Theta_{12} b_{1} \partial_{0}^{2} b_{2}\right)+ \\
\partial_{0}^{2}\left(\Theta_{21} b_{2} \partial_{0}^{1} b_{1}+\Theta_{12} b_{1} \partial_{0}^{1} b_{2}\right) .
\end{array}
$$

Therefore, we have the total-derivative structure yet.

This important property of new perturbation theory is conserved in arbitrary order

over $m$ and $l$ since the time-ordered structure does not depend from upper index of $\hat{j}$, see (C.11).

\section{General formalism of mapping}

The resulting measure looks as follows:

$$
\begin{array}{r}
D M(\xi, \eta)=\frac{1}{\Delta_{c}} \delta\left(E-H_{0}\right) \prod_{t} d^{2} \xi d^{2} \eta \delta\left(\dot{r}_{c}-\frac{\partial H_{j}}{\partial p_{c}}\right) \times \times \\
\delta\left(\dot{p}_{c}+\frac{\partial H_{j}}{\partial r_{c}}\right) \delta\left(\dot{\varphi}_{c}-\frac{\partial H_{j}}{\partial l_{c}}\right) \delta\left(\dot{l}_{c}+\frac{\partial H_{j}}{\partial \varphi_{c}}\right),
\end{array}
$$

Note that the parametrization $\left(r_{c}, p_{c}, \varphi_{c}, l_{c}\right)(\xi, \eta)$ was not specified.

A simple algebra gives:

$$
\begin{array}{r}
D M(\xi, \eta)=\frac{\delta\left(E-H_{0}\right)}{\Delta_{c}} \prod_{t} d^{2} \xi d^{2} \eta \int \prod_{t} d^{2} \bar{\xi} d^{2} \bar{\eta} \times \\
\delta^{2}\left(\bar{\xi}-\left(\dot{\xi}-\frac{\partial h_{j}}{\partial \eta}\right)\right) \delta^{2}\left(\bar{\eta}-\left(\dot{\eta}+\frac{\partial h_{j}}{\partial \xi}\right)\right) \times \\
\delta\left(\frac{\partial r_{c}}{\partial \xi} \cdot \bar{\xi}+\frac{\partial r_{c}}{\partial \eta} \cdot \bar{\eta}+\left\{r_{c}, h_{j}\right\}-\frac{\partial H_{j}}{\partial p_{c}}\right) \times
\end{array}
$$




$$
\begin{array}{r}
\delta\left(\frac{\partial p_{c}}{\partial \xi} \cdot \bar{\xi}+\frac{\partial p_{c}}{\partial \eta} \cdot \bar{\eta}+\left\{p_{c}, h_{j}\right\}+\frac{\partial H_{j}}{\partial r_{c}}\right) \times \\
\delta\left(\frac{\partial \varphi_{c}}{\partial \xi} \cdot \bar{\xi}+\frac{\partial \varphi_{c}}{\partial \eta} \cdot \bar{\eta}+\left\{\varphi_{c}, h_{j}\right\}-\frac{\partial H_{j}}{\partial l_{c}}\right) \times \\
\delta\left(\frac{\partial l_{c}}{\partial \xi} \cdot \bar{\xi}+\frac{\partial l_{c}}{\partial \eta} \cdot \bar{\eta}+\left\{l_{c}, h_{j}\right\}+\frac{\partial H_{j}}{\partial \varphi_{c}}\right) .
\end{array}
$$

The Poisson notation:

$$
\left\{X, h_{j}\right\}=\frac{\partial X}{\partial \xi} \frac{\partial h_{j}}{\partial \eta}-\frac{\partial X}{\partial \eta} \frac{\partial h_{j}}{\partial \xi}
$$

was introduced in (D.2).

We will define the 'auxiliary' quantity $h_{j}$ by following equalities:

$$
\begin{gathered}
\left\{r_{c}, h_{j}\right\}-\frac{\partial H_{j}}{\partial p_{c}}=0,\left\{p_{c}, h_{j}\right\}+\frac{\partial H_{j}}{\partial r_{c}}=0, \\
\left\{\varphi_{c}, h_{j}\right\}-\frac{\partial H_{j}}{\partial l_{c}}=0,\left\{l_{c}, h_{j}\right\}+\frac{\partial H_{j}}{\partial \varphi_{c}}=0 .
\end{gathered}
$$

Then the functional determinant $\Delta_{c}$ is canceled and

$$
D M(\xi, \eta)=\delta\left(E-H_{0}(\eta)\right) \prod_{t} d^{2} \xi d^{2} \eta \delta^{2}\left(\dot{\xi}-\frac{\partial h_{j}}{\partial \eta}\right) \delta^{2}\left(\dot{\eta}+\frac{\partial h_{j}}{\partial \xi}\right),
$$

\section{References}

a) Present address: Lab. of Nuclear Problems, JINR, Dubna, Ru 141980, Russia. E-mail: joseph@nu.jinr.ru

${ }^{1}$ See e.g. R.Rajaraman, Solitons and Instantons (North-Holland Publ. Comp., Amsterdam, New York, Oxford, 1982).

${ }^{2}$ See e.g. M.S.Marinov, Phys.Rep., 60, 1 (1980).

${ }^{3}$ List of solved quantum mechanical problems by time sliced method is given in: C.Grosche, Path Integrals, Hyperbolic Spaces, and Selberg Trace Formulae (World Scint., Singapore, New Jersey, London, Hong Kong, 1995).

${ }^{4}$ See e.g. J.W.Negele, hep-lat/9810053, for review of lattice calculations status in the field theory.

${ }^{5}$ E.g. J.Goldstone and R.Jackiw, Phys.Rev., D11, 1486 (1975); V.E.Korepin and L.D.Faddeev, Theor. Math. Phys., 25, 147 (1975).

${ }^{6}$ The string and $\mathrm{M}$ theories, see references cited e.g. in M.Li, hep-th/9811019, are the important modern examples of a field theory formulation in useful terms to include the nonperturbative effects.

${ }^{7}$ J.Manjavidze, Sov.Nucl.Phys., 45, 442 (1987).

${ }^{8}$ T.W.Kibble, Comm. Math. Phys., 65, 189 (1979).

${ }^{9}$ J.Anandan, Found. Phys., 21, 1265 (1991).

${ }^{10}$ N.P.Konopleva and V.N.Popov, Gauge fields (Harvard Acad. Publ., London-New 
York, 1981).

${ }^{11}$ S.Smale, Inv. Math., 11:1, 45 (1970); R.Abraham and J.E.Marsden, Foundations of Mechanics (Benjamin/ Cummings Publ. Comp., Reading, Mass., 1978).

${ }^{12}$ J.Manjavidze, Perturbation theory in the invariant subspace, hep-th/9801188.

13 R.A.Dashen, B.Hasslacher and A.Neveu, Phys. Rev., D10, 4114 (1977) ${ }^{14}$ V.Fock, Vestnik LGU, 16, 442 (1959).

${ }^{15}$ R.Mills, Propagators of Many-Particles Systems, (Gordon \& Breach, 1969).

${ }^{16}$ V.I.Arnold,Mathematical Methods of Classical Mechanics,(Springer Verlag, New York, 1978).

17 The connection between space-time metrics and symmetries one can note comparing classical solutions of Yang-Mills equations listed in A.Actor, Rev. Mod. Phys., 51, 461 (1979).

${ }^{18}$ V.S.Popov, High Energy Physics and Elementary Particles Theory, (Naukova Dumka, Kiev, 1967).

${ }^{19}$ I.H.Duru and H.Kleinert, Phys. Lett., B84, 185 (1979). 